\title{
Neutrino quasinormal modes of the Reissner-Nordström black hole
}

\author{
Jiliang Jing* \\ Institute of Physics and Department of Physics, \\ Hunan Normal University, \\ Changsha, Hunan 410081, P. R. China
}

\begin{abstract}
The neutrino quasinormal modes of the Reissner-Nordström (RN) black hole are investigated using continued fraction approach. We find, for large angular quantum number, that the quasinormal frequencies become evenly spaced and the spacing of the real part depends on the charge of the black hole and that of the imaginary part is zero. We then find that the quasinormal frequencies in the complex $\omega$ plane move counterclockwise as the charge increases. They get a spiral-like shape, moving out of their Schwarzschild value and "looping in" towards some limiting frequency as the charge tends to the extremal value. The number of the spirals increases as the overtone number increases but it decreases as the angular quantum number increases. We also find that both the real and imaginary parts are oscillatory functions of the charge, and the oscillation becomes faster as the overtone number increases but it becomes slower as the angular quantum number increases.
\end{abstract}

KEYwords: Quasinormal modes, Neutrino, Reissner-Norström black hole.

\footnotetext{
*Electronic address: jljing@hunnu.edu.cn
} 


\section{Contents}

1. introduction 1

2. Neutrino equations in the Reissner-Nordström spacetime 2

3. Numerical Approaches 4

4. Neutrino Quasinormal Modes of the Reissner-Nordström black hole 5

4.1 Fundamental quasinormal frequencies 5

4.2 QNMs for large angular quantum number 5

4.3 Highly damped modes 6

5. summary 7

\section{1. introduction}

It is well-known that the quasinormal frequencies of a black hole are defined as proper solutions of the perturbation equations belonging to certain complex characteristic frequencies which satisfy the boundary conditions appropriate for purely ingoing waves at the event horizon and purely outgoing waves at infinity [1]. The quasinormal modes (QNMs) are entirely fixed by the structure of the background spacetime and are irrelevant of the initial perturbations [1]. Thus, it is generally believed that QNMs carry a unique footprint to directly identify the existence of a black hole. Through the QNMs, one can extract information about the physical parameters of the black hole - mass, electric charge, and angular momentum - from the gravitational wave signal by fitting the few lowest observed quasinormal frequencies to those calculated from the perturbation analysis. Meanwhile, the study of QNMs may lead to a deeper understanding of the thermodynamic properties of black holes in loop quantum gravity [2] [3], as well as the QNMs of anti-de Sitter black holes have a direction interpretation in terms of the dual conformal field theory [4] [5] [6]. Therefore, much attention has been devoted to the study of the quasinormal modes in the recent thirty years [7]-21].

Although QNMs of scalar, electromagnetic and gravitational perturbations are studied extensively, the investigation of the QNMs of the Dirac field is very limited [22] 223] [24]. Furthermore, in these papers the study of the Dirac QNMs were limited to use WKB or Pöshl-Teller potential approximative methods. The WKB and Pöshl-Teller potential approximative methods may be valuable tools in certain situations, but in general results obtained from the approximative methods should not be expected to be accurate. It is well known that the Leaver's continued fraction technique is the best workhorse method to compute highly damped modes and is very reliable. However, the method can not be directly used to study the QNMs of the Dirac fields if we take the standard wave equations. The reason to obstruct the study of the Dirac QNMs using continued fraction technique is that for static black holes the standard wave equation

$$
\left(\frac{d^{2}}{d r_{*}^{2}}+\omega^{2}\right) Z_{ \pm}=V_{ \pm} Z_{ \pm}
$$


possesses a special potential

$$
V_{ \pm}=\lambda^{2} \frac{\Delta}{r^{4}} \pm \lambda \frac{d}{d r_{*}} \frac{\sqrt{\Delta}}{r^{2}}
$$

which is the function of $\sqrt{\Delta}$ [22] [24], where $\Delta$ is a function related to the metric, say $\Delta=$ $r^{2}-2 M r+Q^{2}$ for the RN black hole. We can not investigate the Dirac QNMs using the continued fraction approaches because we have to expand the potential as a series at the event horizon but the factor $\sqrt{\Delta}$ does not permit us to do that.

Recently, we 25] found that the wave function and potential of the Dirac field can be expressed as new forms, and the new wave function are related to the standard one in a simple way [25]. Starting from the new wave function and potential, we [25, 26] studied that the Dirac QNMs of the Schwarzschild black hole using continued fraction [27] and Hill-determinant approaches [28] 29] and the Dirac QNMs of the Schwarzschild-anti-de Sitter and Reissner-Nordström-anti-de Sitter black

holes using Horowitz-Hubeny approach [16]. Considering the RN black hole spacetime provides a better background than the Schwarzschild geometry, in this paper we will extend the study in Ref. 25] to the RN black hole, and to see how the quasinormal frequencies depend on the charge of the black hole.

The organization of this paper is as follows. In Sec. 2 the decoupled neutrino equations and corresponding wave equation in the RN spacetime are obtained using Newman-Penrose formalism. In Sec. 3 the numerical approach to compute the neutrino QNMs is introduced. In Sec. 4 the numerical results for the neutrino QNMs in the RN black hole are presented. The last section is devoted to a summary.

\section{Neutrino equations in the Reissner-Nordström spacetime}

The neutrino equations [30] can be expressed as

$$
\begin{aligned}
& \sqrt{2} \nabla_{B B^{\prime}} P^{B}=0, \\
& \sqrt{2} \nabla_{B B^{\prime}} Q^{B}=0,
\end{aligned}
$$

where $\nabla_{B B^{\prime}}$ is covariant differentiation, $P^{B}$ and $Q^{B}$ are the two-component spinors representing the wave functions, $\bar{P}_{B^{\prime}}$ is the complex conjugate of $P_{B}$. In the Newman-Penrose formalism [31] the equations become

$$
\begin{aligned}
& (D+\epsilon-\rho) P^{0}+(\bar{\delta}+\pi-\alpha) P^{1}=0 \\
& (\triangle+\mu-\gamma) P^{1}+(\delta+\beta-\tau) P^{0}=0 \\
& (D+\bar{\epsilon}-\bar{\rho}) \bar{Q}^{0^{\prime}}+(\delta+\bar{\pi}-\bar{\alpha}) \bar{Q}^{1^{\prime}}=0 \\
& (\triangle+\bar{\mu}-\bar{\gamma}) \bar{Q}^{1^{\prime}}+(\bar{\delta}+\bar{\beta}-\bar{\tau}) \bar{Q}^{0^{\prime}}=0
\end{aligned}
$$

The null tetrad for the RN black hole can be taken as

$$
\begin{aligned}
l^{\mu} & =\left(\frac{r^{2}}{\Delta}, 1,0,0\right), \\
n^{\mu} & =\frac{1}{2}\left(1,-\frac{\Delta}{r^{2}}, 0,0\right) \\
m^{\mu} & =\frac{1}{\sqrt{2} r}\left(0,0,1, \frac{i}{\sin \theta}\right),
\end{aligned}
$$


with

$$
\Delta=r^{2}-2 M r+Q^{2}=\left(r-r_{+}\right)\left(r-r_{-}\right),
$$

where $M$ and $Q$ are the mass and charge of the RN black hole, $r_{+}=M+\sqrt{M^{2}-Q^{2}}$ and $r_{-}=M-\sqrt{M^{2}-Q^{2}}$ represent the event and inner horizons. The wave functions can be expressed as

$$
\begin{aligned}
P^{0} & =\frac{1}{r} \mathbb{R}_{-1 / 2}(r) S_{-1 / 2}(\theta) e^{-i(\omega t-\bar{m} \varphi)}, \\
P^{1} & =\mathbb{R}_{+1 / 2}(r) S_{+1 / 2}(\theta) e^{-i(\omega t-\bar{m} \varphi)} \\
\bar{Q}^{1^{\prime}} & =\mathbb{R}_{+1 / 2}(r) S_{-1 / 2}(\theta) e^{-i(\omega t-\bar{m} \varphi)}, \\
\bar{Q}^{0^{\prime}} & =-\frac{1}{r} \mathbb{R}_{-1 / 2}(r) S_{+1 / 2}(\theta) e^{-i(\omega t-\bar{m} \varphi)},
\end{aligned}
$$

where $\omega$ and $\bar{m}$ are the energy and angular momentum of the neutrino particle. After the tedious calculation Eq. (2.2) can be simplified as

$$
\begin{aligned}
& \sqrt{\Delta} \mathcal{D}_{0} \mathbb{R}_{-1 / 2}=\lambda \sqrt{\Delta} \mathbb{R}_{+1 / 2}, \\
& \sqrt{\Delta} \mathcal{D}_{0}^{\dagger}\left(\sqrt{\Delta} \mathbb{R}_{+1 / 2}\right)=\lambda \mathbb{R}_{-1 / 2}, \\
& \mathcal{L}_{1 / 2} S_{+1 / 2}=-\lambda S_{-1 / 2}, \\
& \mathcal{L}_{1 / 2}^{\dagger} S_{-1 / 2}=\lambda S_{+1 / 2},
\end{aligned}
$$

with

$$
\begin{aligned}
& \mathcal{D}_{n}=\frac{\partial}{\partial r}-\frac{i r^{2} \omega}{\Delta}+\frac{n}{\Delta} \frac{d \Delta}{d r}, \\
& \mathcal{D}_{n}^{\dagger}=\frac{\partial}{\partial r}+\frac{i r^{2} \omega}{\Delta}+\frac{n}{\Delta} \frac{d \Delta}{d r}, \\
& \mathcal{L}_{n}=\frac{\partial}{\partial \theta}+\frac{m}{\sin \theta}+n \cot \theta \\
& \mathcal{L}_{n}^{\dagger}=\frac{\partial}{\partial \theta}-\frac{m}{\sin \theta}+n \cot \theta
\end{aligned}
$$

where $\lambda= \pm\left(l+\frac{1}{2}\right)$ ( $l$ is the quantum number characterizing the angular distribution). We will focus our attention on the massless Dirac field in this paper. Therefore, we can eliminate $\mathbb{R}_{-1 / 2}$ (or $\sqrt{\Delta} \mathbb{R}_{+1 / 2}$ ) from Eqs. (2.6) and (2.7) to obtain a radial decoupled Dirac equation for $\sqrt{\Delta} \mathbb{R}_{+1 / 2}$ (or $\mathbb{R}_{-1 / 2}$ ). Then, introducing an usual tortoise coordinate

$$
d r_{*}=\frac{r^{2}}{\Delta} d r
$$

and resolving the equation in the form

$$
\mathbb{R}_{s}=\frac{\Delta^{-s / 2}}{r} \Psi_{s}
$$

we obtain the wave equation

$$
\frac{d^{2} \Psi_{s}}{d r_{*}^{2}}+\left(\omega^{2}-V_{s}\right) \Psi_{s}=0
$$

with

$$
V_{s}=-\frac{\Delta}{4 r^{2}} \frac{d}{d r}\left[r^{2} \frac{d}{d r}\left(\frac{\Delta}{r^{4}}\right)\right]+\frac{s^{2} r^{4}}{4}\left[\frac{d}{d r}\left(\frac{\Delta}{r^{4}}\right)\right]^{2}+i s \omega r^{2} \frac{d}{d r}\left(\frac{\Delta}{r^{4}}\right)+\frac{\lambda^{2} \Delta}{r^{4}} .
$$

We will study the neutrino quasinormal frequencies of the RN black hole using Eqs. (2.13) and (2.14) and with the help of the continued fraction approach. 


\section{Numerical Approaches}

For the RN black hole, the QNMs are defined to be modes with purely ingoing waves at the event horizon and purely outgoing waves at infinity [1]. Then, the boundary conditions on wave function $\Psi_{s}$ at the horizon $\left(r=r_{+}\right)$and infinity $(r \rightarrow+\infty)$ can be expressed as

$$
\Psi_{s} \sim \begin{cases}\left(r-r_{+}\right)^{-\frac{s}{2}-\frac{i \omega}{2 \kappa_{+}}} & r \rightarrow r_{+} \\ r^{-s+i \omega} e^{i \omega r} & r \rightarrow+\infty\end{cases}
$$

where $\kappa_{+}=\frac{r_{+}-r_{-}}{2 r_{+}^{2}}$ is the surface gravity on the event horizon $r_{+}$.

A solution to Eq. (2.13) that has the desired behavior at the boundary can be written in the form

$$
\Psi_{s}=r\left(r-r_{+}\right)^{-\frac{s}{2}-\frac{i \omega}{2 \kappa_{+}}}\left(r-r_{-}\right)^{-1-\frac{s}{2}+2 i \omega+\frac{i \omega}{2 \kappa_{-}}} e^{i \omega\left(r-r_{-}\right)} \sum_{m=0}^{\infty} a_{m}\left(\frac{r-r_{+}}{r-r_{-}}\right)^{m}
$$

where $\kappa_{-}=\frac{r_{+}-r_{-}}{2 r_{-}^{2}}$ is the surface gravity on the inner horizon $r_{-}$. If we take $r_{+}+r_{-}=1$ and $b=r_{+}-r_{-}$, the sequence of the expansion coefficients $\left\{a_{m}: m=1,2, \ldots\right\}$ is determined by a three-term recurrence relation staring with $a_{0}=1$ :

$$
\begin{aligned}
& \alpha_{0} a_{1}+\beta_{0} a_{0}=0, \\
& \alpha_{m} a_{m+1}+\beta_{m} a_{m}+\gamma_{m} a_{m-1}=0, \quad m=1,2, \ldots
\end{aligned}
$$

The recurrence coefficient $\alpha_{m}, \beta_{m}$ and $\gamma_{m}$ are given in terms of $m$ and the black hole parameters by

$$
\begin{aligned}
& \alpha_{m}=m^{2}+\left(C_{0}+1\right) m+C_{0}, \\
& \beta_{m}=-2 m^{2}+\left(C_{1}+2\right) m+C_{3}, \\
& \gamma_{m}=m^{2}+\left(C_{2}-3\right) m+C_{4}-C_{2}+2,
\end{aligned}
$$

and the intermediate constants $C_{m}$ are defined by

$$
\begin{aligned}
& C_{0}=1-s-i \omega-\frac{i \omega\left(r_{+}^{2}+r_{-}^{2}\right)}{b}, \\
& C_{1}=-4+2 i \omega(2+b)+\frac{2 i \omega\left(r_{+}^{2}+r_{-}^{2}\right)}{b} \\
& C_{2}=s+3-3 i \omega-\frac{i \omega\left(r_{+}^{2}+r_{-}^{2}\right)}{b} \\
& C_{3}=\omega^{2}\left(4+2 b-4 r_{+} r_{-}\right)-s-1+(2+b) i \omega-\lambda^{2}+(2 \omega+i) \frac{\omega\left(r_{+}^{2}+r_{-}^{2}\right)}{b}, \\
& C_{4}=s+1-2 \omega^{2}-(2 s+3) i \omega-(2 \omega+i) \frac{\omega\left(r_{+}^{2}+r_{-}^{2}\right)}{b} .
\end{aligned}
$$

It is interesting to note that the three-term recursion relation is obtained form the wave equation (2.13) directly. The calculation is simpler than other cases. For example, the coefficients of the expansion for the electromagnetic and gravitational fields in the RN black hole are determined by a four-term recursion relation which should be reduced to a three-term relation using a Gaussian eliminated step (see [29] for details). 
The series in (3.2) converges and the $r=+\infty$ boundary condition (3.1) is satisfied if, for a given $s$ and $\lambda$, the frequency $\omega$ is a root of the continued fraction equation

$$
\left[\beta_{m}-\frac{\alpha_{m-1} \gamma_{m}}{\beta_{m-1}-} \frac{\alpha_{m-2} \gamma_{m-1}}{\beta_{m-2}-} \ldots \frac{\alpha_{0} \gamma_{1}}{\beta_{0}}\right]=\left[\frac{\alpha_{m} \gamma_{m+1}}{\beta_{m+1}-} \frac{\alpha_{m+1} \gamma_{m+2}}{\beta_{m+2}-} \frac{\alpha_{m+2} \gamma_{m+3}}{\beta_{m+3}-} \ldots\right], \quad(m=1,2 \ldots) .
$$

This leads to a simple method to find quasinormal frequencies - defining a function which returns the value of the continued fraction for an initial guess at the frequency, and then use a root finding routine to find the zeros of this function in the complex plane. The frequency for which happens is a quasinormal frequency. The $n$th quasinormal frequency is usually found to be the most stable root of the $n$th inversion 27].

\section{Neutrino Quasinormal Modes of the Reissner-Nordström black hole}

In this section we will present the numerical results of the neutrino quasinormal frequencies of the RN black hole obtained by using the numerical approach just outlined in the previous section. The results will be organized into three subsections: the fundamental quasinormal frequencies, the QNMs for large angular quantum number and highly damped modes.

\subsection{Fundamental quasinormal frequencies}

We find that the change of the neutrino quasinormal frequencies becomes faster as the charge increases. Therefore, as we approach the extremal value $Q=1 / 2$, the convergence of the continued fraction method slower, and the required computing time gets longer. The fundamental neutrino quasinormal frequencies of the $\mathrm{RN}$ black hole for $Q=0$ to $Q=0.499$ and $\lambda=1$ are listed in the table I. We know from the table that the real part of the fundamental quasinormal frequency increases as $Q$ increases, but the imaginary part decreases first and then increases as $Q$ increases (we should note that the magnitude of the imaginary part increases first and then decreases). It is shown that the intermediate decay of the Dirac perturbation around the RN black hole depends on the charge $Q$. The oscillating frequency of this decay which corresponds to $\omega_{R}$ increases as $Q$ increases, and the amplitude per unit time $\sim e^{\omega_{I}}$ increases first and then decreases. We should point out that this property is true of all fundamental quasinormal frequencies with different angular quantum number.

\subsection{QNMs for large angular quantum number}

The quasinormal frequencies for $\lambda=1$ to $\lambda=40$ and $n=0,1,2$ are described by Fig. 1 which shows that $\Delta \omega=\omega_{\lambda+1}-\omega_{\lambda}$ as a function of $\lambda$. From the figure we know that, for the cases of $n=0, n=1$ and $n=2$, the quasinormal frequencies become evenly spaced for large $\lambda$ and the spacing is given by

$$
\begin{aligned}
& \Delta \omega(Q=0.0)=0.38490-0.0000 i . \\
& \Delta \omega(Q=0.1)=0.38750-0.0000 i . \\
& \Delta \omega(Q=0.2)=0.39581-0.0000 i . \\
& \Delta \omega(Q=0.3)=0.41170-0.0000 i .
\end{aligned}
$$

That is to say, the spacing of the real part is related to the charge $Q$ and the spacing of the imaginary part becomes zero for large $\lambda$. 


\begin{tabular}{c|c|c|c|c|c}
\hline \hline$Q$ & $\omega$ & $Q$ & $\omega$ & $Q$ & $\omega$ \\
\hline 0.000 & $0.365926-0.193965 \mathrm{i}$ & 0.410 & $0.427193-0.196008 \mathrm{i}$ & 0.460 & $0.452381-0.190363 \mathrm{i}$ \\
0.050 & $0.366591-0.194066 \mathrm{i}$ & 0.415 & $0.429341-0.195734 \mathrm{i}$ & 0.465 & $0.455387-0.189224 \mathrm{i}$ \\
0.100 & $0.368615-0.194366 \mathrm{i}$ & 0.420 & $0.431562-0.195418 \mathrm{i}$ & 0.470 & $0.458469-0.187904 \mathrm{i}$ \\
0.150 & $0.372096-0.194851 \mathrm{i}$ & 0.425 & $0.433862-0.195052 \mathrm{i}$ & 0.475 & $0.461608-0.186375 \mathrm{i}$ \\
0.200 & $0.377208-0.195488 \mathrm{i}$ & 0.430 & $0.436242-0.19463 \mathrm{i}$ & 0.480 & $0.464769-0.184605 \mathrm{i}$ \\
0.250 & $0.384241-0.196213 \mathrm{i}$ & 0.435 & $0.438707-0.194144 \mathrm{i}$ & 0.485 & $0.467892-0.182571 \mathrm{i}$ \\
0.300 & $0.393651-0.196891 \mathrm{i}$ & 0.440 & $0.441258-0.193585 \mathrm{i}$ & 0.490 & $0.47089-0.180287 \mathrm{i}$ \\
0.350 & $0.406184-0.19722 \mathrm{i}$ & 0.445 & $0.4439-0.192942 \mathrm{i}$ & 0.495 & $0.473689-0.177851 \mathrm{i}$ \\
0.400 & $0.423108-0.196443 \mathrm{i}$ & 0.450 & $0.446635-0.192202 \mathrm{i}$ & 0.497 & $0.474767-0.176866 \mathrm{i}$ \\
0.405 & $0.425116-0.196243 \mathrm{i}$ & 0.455 & $0.449462-0.191348 \mathrm{i}$ & 0.499 & $0.475833-0.175873 \mathrm{i}$ \\
\hline \hline
\end{tabular}

Table 1: The fundamental neutrino quasinormal frequencies of the $\mathrm{RN}$ black hole for $Q=0$ to $Q=0.499$ and $\lambda=1$. As charge $Q$ increases, the real part of the fundamental quasinormal frequency increases, but the imaginary part decreases first and then increases.
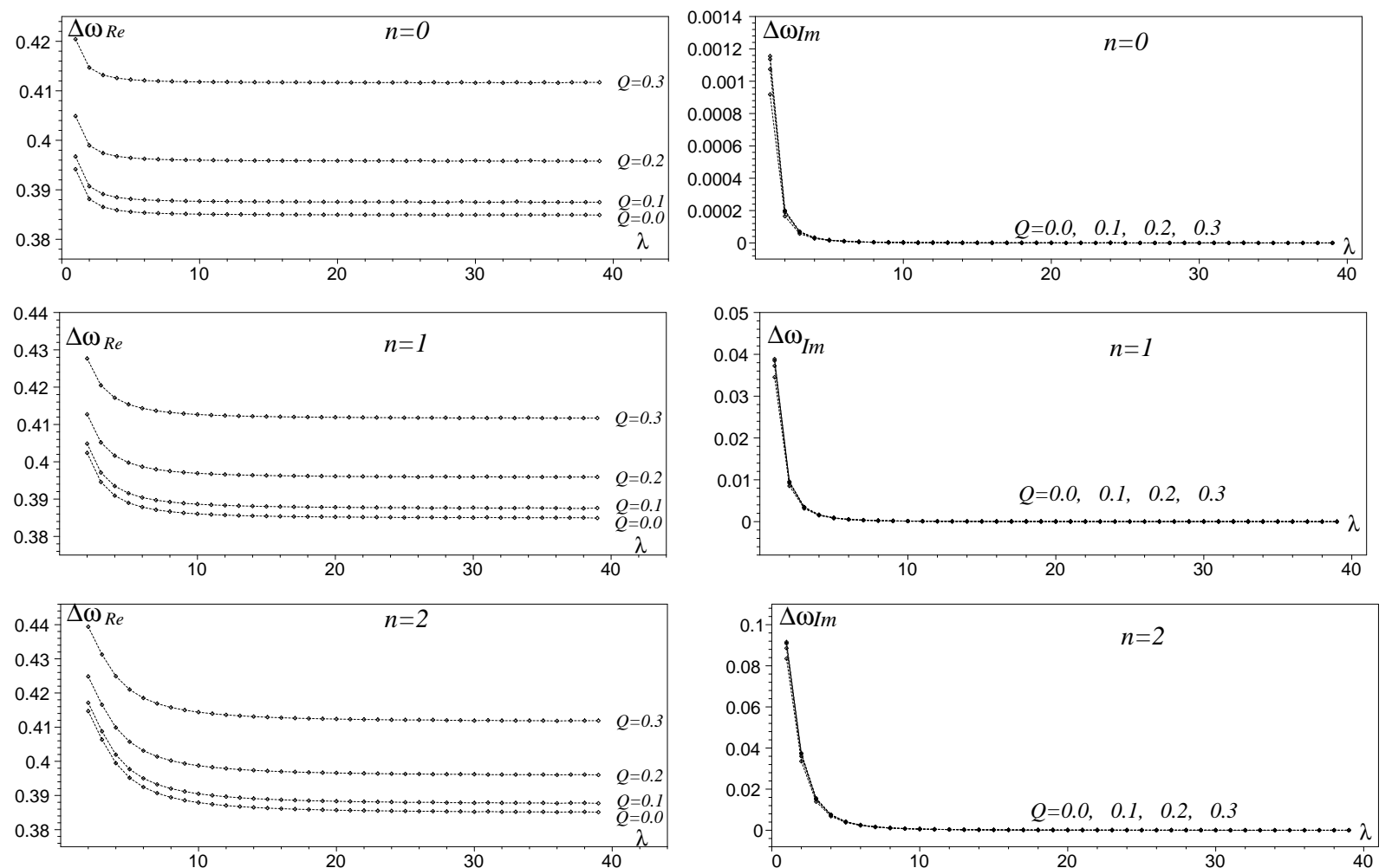

Figure 1: The spacing $\Delta \omega=\omega_{\lambda+1}-\omega_{\lambda}$ as the functions of $\lambda$ for $n=0,1,2$ quasinormal frequencies. The left three figures are drawn for $\Delta \omega_{R e}=\operatorname{Re}\left(\omega_{\lambda+1}\right)-\operatorname{Re}\left(\omega_{\lambda}\right)$ which show that the spacing of the real part is related to the charge $Q$ for large $\lambda$. The right three figures are for $\Delta \omega_{\operatorname{Im}}=\operatorname{Im}\left(\omega_{\lambda+1}\right)-\operatorname{Im}\left(\omega_{\lambda}\right)$ which show that the spacing of the imaginary part becomes zero for large $\lambda$.

\subsection{Highly damped modes}

The Fig. 2 is drawn for $n=0$ to $n=3$ and $\lambda=1$, and Fig. 3 is for $n=5,6,7,8,10$ and $\lambda=2$. In these figures the quasinormal frequency trajectories are formed only by points which are quasinormal frequencies at increments in $Q$ of 0.01 for $Q=0$ to $Q \sim 0.40$ and at increments in $Q$ of 0.001 for $Q \sim 0.40$ to $Q=0.495$. We now proceed to discussed the figures in more detail. 
The left columns in the Figs. 2 and 3 describe the behavior of quasinormal frequencies in the complex $\omega$ plane which show that the frequencies generally move counterclockwise as the charge increases. They get a spiral-like shape, moving out of their Schwarzschild value $(Q=0)$ and "looping in" towards some limiting frequency as the charge $Q$ tends to the extremal value, $Q=1 / 2$. For a given $\lambda$, we observe that the number of spirals increases as the overtone number increases. However, for a given overtone number $n$, increasing $\lambda$ has the effect of "unwinding" the spirals, as we see in the two figures that the spiral begins at $n=2$ for $\lambda=1$ but it starts at $n=6$ for $\lambda=2$.

The second and last columns in the Figs. 2 and 3 illustrate that the real and imaginary parts of the quasinormal frequencies are the functions of the charge $Q$. We know from these figures that both the real and imaginary parts of the frequencies are oscillatory functions of the charge. The oscillation starts earlier and earlier as the overtone number $n$ grows for a fixed $\lambda$, but it begins later and later as the angular quantum number increases for a fixed $n$. Meanwhile, the oscillation becomes faster as the overtone number increases for a given $\lambda$, but it becomes slower as $\lambda$ increases for a given $n$.

\section{5. summary}

The wave equation for the neutrino fields in the RN black hole spacetime is obtained by means of the Newman-Penrose formulism. The expansion coefficients of the wave equation satisfying appropriate boundary conditions are determined by a three-term recurrence relation. Then, the neutrino quasinormal frequencies of the RN black hole spacetime are evaluated using continued fraction approach and the results are presented by table and figures. We find, for any angular quantum number, that the real part of the fundamental quasinormal frequency increases as the charge increases, but the imaginary part decreases first and then increases as the charge increases. The fact tell us that the intermediate decay of the Dirac perturbation around the RN black hole depends on the charge. As the charge increases, the oscillating frequency of this decay increases and the amplitude per unit time increases first and then decreases. We then show that the quasinormal frequencies become evenly spaced for large $\lambda$ and the spacing of the real part is related to the charge and that of the imaginary part becomes zero. We also find that the frequencies in the complex $\omega$ plane generally move counterclockwise as the charge is increased. They get a spiral-like shape, moving out of their Schwarzschild value and "looping in" towards some limiting frequency as the charge tends to the extremal value. For a given $\lambda$, we observe that the number of spirals increases as the overtone number increases. However, for a given overtone number, increasing $\lambda$ has the effect of "unwinding" the spirals. At last, we find that the real and imaginary parts of the quasinormal frequencies are oscillatory functions of charge, and the oscillating behavior starts earlier and earlier as the overtone number grows for a fixed $\lambda$, but it begins later and later as $\lambda$ increases for a fixed overtone number.

\section{Acknowledgments}

This work was supported by the National Natural Science Foundation of China under Grant No. 10473004; the FANEDD under Grant No. 200317; the SRFDP under Grant No. 20040542003; the Hunan Provincial Natural Science Foundation of China under Grant No. 05JJ0001. 

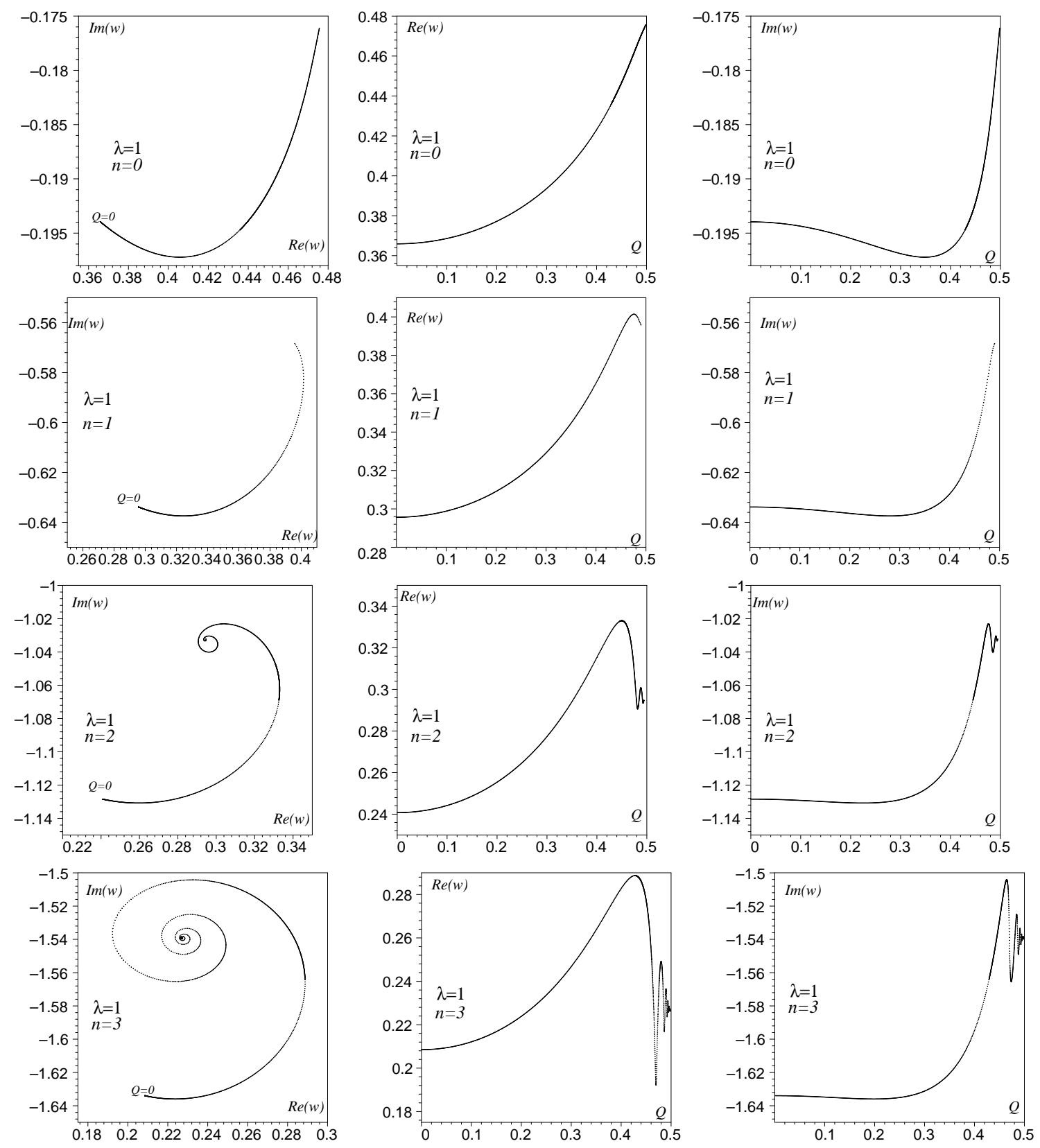

Figure 2: The left 4 figures describe the behavior of quasinormal frequencies for $n=0$ to $n=3$ and $\lambda=1$ in the complex $\omega$ plane which show that the frequencies generally move counterclockwise as the charge is increased and the number of spirals increases as the overtone number increases. The others graph $\operatorname{Re}(\omega)$ and $\operatorname{Im}(\omega)$ of the quasinormal frequencies versus charge $Q$ which tell us that both the real and imaginary parts are oscillatory functions of charge and the oscillations become faster as the overtone number increases.

\section{References}

[1] S. Chandrasekhar and S. Detweller, Proc. R. Soc. Lond. A 344, 441 (1975).

[2] S. Hod, Phys. Rev. Lett. 81, 4293 (1998).

[3] O. Dreyer, Phys. Rev. Lett. 90, 081301 (2003).

[4] J. Maldacena, Adv. Theor. Math. Phys. 2, 231 (1998).

[5] E. Witten, Adv. Theor. Math. Phys. 2, 253 (1998). 
[6] S. Kalyana Rama and Sathiapalan, Mod Phys. Lett. A 14, 2635 (1999).

[7] J. S. F. Chan and R. B. Mann, Phys. Rev. D 55, 7546 (1997).

[8] D. Birmingham, I. Sachs, and S. N. Solodukhin, Phys. Rev. Lett. 88, 151301 (2002).

[9] T. Regge and J. A. Wheeler, Phys. Rev. 108, 1063 (1957).

[10] K. Kokkotas and B. Schmidt, Living Reviews Relativ. 2, 2 (1999).

[11] H. P. Nollert, Class. Quant. Grav. 16, 159 (1999).

[12] Songbai Chen and Jiliang Jing, Class. Quant. Grav. 22, 533 (2005); ibid. 22, 1129 (2005).

[13] I. G. Moss and J. P. Norman, Class. Quant. Grav. 19, 2323 (2002).

[14] M. Giammatteo and Ian G. Moss, Class. Quant. Grav. 22, 1803 (2005).

[15] A. O. Starinets, Phys. Rev. D 66, 124013 (2002).

[16] G. T. Horowitz and V. E. Hubeny, Phys. Rev. D 62, 024027 (2000).

[17] Y. Kurita and M. A. Sakagami, Phys. Rev. D 67, 024003 (2003).

[18] V. Cardoso and J. P. S. Lemos, Phys. Rev. D 64, 084017 (2001); V. Cardoso, R. Konoplya, and J. P. S. Lemos, gr-qc/0305037; V. Cardoso and J. P. S. Lemos, Phys. Rev. D 63, 124015 (2001).

[19] E. Berti and K. D. Kokkotas, hep-th/0303029.

[20] H. Onozawa, T. Mishima, T. Okamura, and H. Ishihara, Phys. Rev. D 53, 7033 (1996).

[21] R. A. Konoplya, Phys. Rev. D 66, 044009 (2002).

[22] H. T. Cho, Phys. Rev. D 68, 024003 (2003).

[23] A. Zhidenko, Class. Quant. Grav. 21, 273 (2004).

[24] Jiliang Jing, Phys. Rev. D 69, 084009 (2004); D 70, 065004 (2004).

[25] Jiliang Jing, Phys. Rev. D 71, 124006 (2005).

[26] Jiliang Jing, Phys. Rev. D 71, 124011 (2005).

[27] E. W. Leaver, Pro. R. Soc. Lond. A, 402, 285 (1985); E. W. Leaver, Phys. Rev. D 34, 384 (1986).

[28] B. Majumdar and N. Panchapakesan, Phys. Rev. D 40, 2568 (1989).

[29] E. W. Leaver, Phys. Rev. D 41, 2986 (1990).

[30] D. N. Page, Phys. Rev. D 14, 1509 (1976).

[31] E. Newman and R. Penrose, J. Math. Phys. (N. Y.) 3, 566 (1962). 

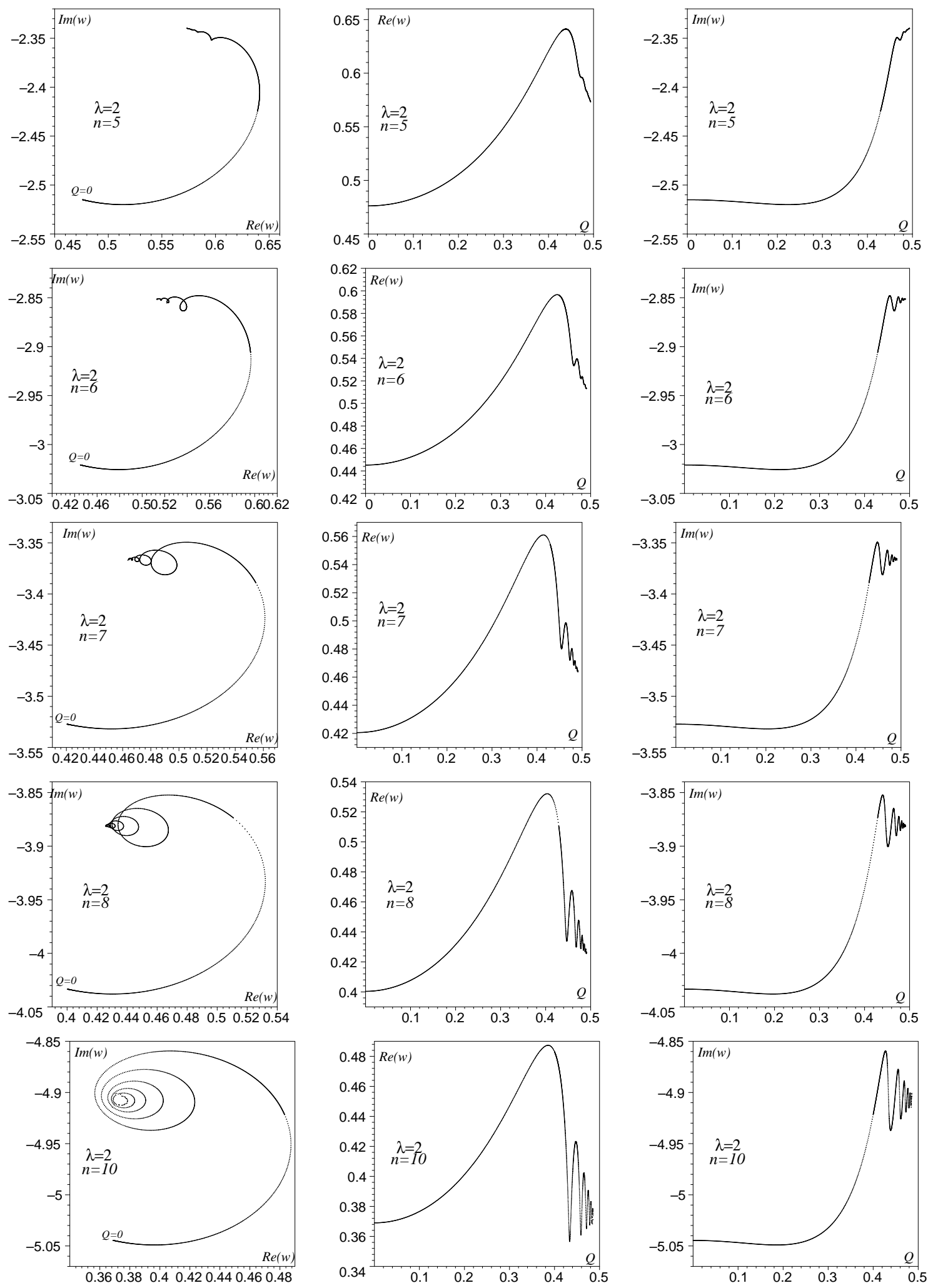

Figure 3: The left 5 figures describe the behavior of quasinormal frequencies for $n=5,6,7,8,10$ and $\lambda=2$ in the complex $\omega$ plane and the others graph $\operatorname{Re}(\omega)$ and $\operatorname{Im}(\omega)$ of the quasinormal frequencies versus charge $Q$. By comparing it with Fig. 2 we know that the number of the spirals decreases and the oscillations become slower as the angular quantum number increases. 